\title{
EVALUACIÓN FORMATIVA Y CLASE INVERTIDA PARA LA ADQUISICIÓN DE COMPETENCIAS EN EL MÁSTER DE PROFESORADO DE EDUCACIÓN SECUNDARIA
}

Formative assessment and flipped classroom for the acquisition of competences in in the Unviersity Máster in Secondary

Avaliação formativa e aula invertida para a aquisição de competências no mestrado de formação de professores do ensino secundário

\author{
Nuria Ureña Ortín (1) \\ Pedro A. López Miñarro (2)
}

Este estudio se ha llevado a cabo dentro del Grupo Innovación Docente metodologías activas, TICS y evaluación en el área de Didáctica de la Expresión Corporal y Musical (Grupo MATE)

(1) Universidad de Murcia, España. Teléfono: +34 86888 8470. Correo electrónico: nuriaur@um.es

(2) Universidad de Murcia, España. Teléfono: +34 86888 7051. Correo electrónico: palopez@um.es

\section{Resumen}

El objetivo de esta experiencia fue aplicar un proceso de evaluación formativa y compartida unido a una metodología de clase invertida. Este planteamiento exige igualmente organizar el proceso de enseñanza-aprendizaje teniendo como referencia las competencias profesionales. La muestra estuvo constituida por 23 estudiantes matriculados en la asignatura Evaluación y Recursos en la Enseñanza de la Educación Física del Máster de formación de Profesorado durante el curso 2018/19. En general la experiencia de buena práctica analizada resultó eficaz, tanto para el alumnado como el profesorado. En cuanto al alumnado: (1) se han obtenido muy buenos resultados académicos debido a la mayor retroalimentación recibida en las tareas a realizar; (2) En las sesiones presenciales se garantizó una participación activa del estudiante y se generaban debates de calidad. En cuanto a la docente ha percibido una mayor motivación y calidad en los trabajos realizados por parte del alumnado.

Palabras clave: Evaluación formativa; evaluación compartida; master de formación de profesorado; flipped classroom 


\begin{abstract}
The objective of this experience was to apply a formative and shared evaluation process together with an inverted class methodology. This approach also requires organizing the teaching-learning process with reference to professional skills. The sample consisted of 23 students enrolled in the subject Evaluation and Resources in the Teaching of Physical Education of the Master's Degree in Teacher Training during the 2018/19 academic year. In general, the experience of good practice analyzed was effective, both for students and teachers. Regarding the students: (1) very good academic results have been obtained due to the greater feedback received in the tasks to be carried out; (2) In the face-to-face sessions, an active participation of the student was guaranteed and quality debates were generated; As for the teacher, she has perceived greater motivation and quality in the work done by the students
\end{abstract}

Keywords: Formative assessment shared evaluation; teacher training course; flipped classroom

\title{
Resumo
}

O objetivo desta experiência foi aplicar um processo de avaliação formativa e partilhada, juntamente com uma metodologia de aula invertida. Esta abordagem requer também, a organização do processo de ensino-aprendizagem, tendo como referência as competências profissionais. A amostra foi composta por 23 alunos matriculados na disciplina Avaliação e Recursos no Ensino da Educação Física do Mestrado de Formação de Professores durante o ano letivo 2018/19. De uma forma geral, a experiência de boas práticas analisada foi eficaz tanto para os alunos como para os professores. Em relação aos alunos: (1) obtiveram-se resultados escolares muito bons devido ao maior feedback recebido nas tarefas a realizar; (2) Nas sessões presenciais, garantiu-se uma participação ativa do aluno e geraram-se debates de qualidade. Em relação à professora, apercebeu-se de uma maior motivação e qualidade no trabalho realizado pelos alunos.

Palavras-chave: Avaliação formativa; avaliação partilhada; mestrado de formação de professores; aula invertida 


\section{Introducción}

Esta experiencia floreció a partir de la siguiente cuestión ¿Cómo puede un docente conseguir que los estudiantes sean activos en las clases, más autónomos en sus aprendizajes y reflexivos sobre el proceso de aprendizaje adquirido? El primer paso sería establecer unos pilares de actuación docente que permita orientar el proceso de enseñanza hacia el alumnado transformando el uso que se hace tradicionalmente de la evaluación.

En este trabajo se apuesta, en primer lugar (primer pilar), por aplicar una evaluación formativa y compartida (Barrientos, López-Pastor \& Pérez Pueyo, 2019; López Pastor \& Pérez Pueyo, 2017) donde el alumnado tenga la posibilidad de intervenir en el proceso de evaluación y en la toma de decisiones, tanto en los aspectos relacionados con la evaluación como en la calificación (Herranz \& López-Pastor, 2017). Igualmente, la evaluación se va a utilizar como un procedimiento de tutorización y seguimiento del proceso de aprendizaje del alumnado (Perez-Pueyo et al., 2019).

Para conseguir que el estudiante sea un agente activo en las clases, la metodología se convierte en el segundo elemento clave (segundo pilar). De las innumerables metodologías activas se ha elegido flipped classroom (clase invertida). Supone básicamente aprovecharse de las potencialidades que presentan las TIC para acercar al alumnado los recursos necesarios que pueden ser consultados fuera del aula, y aprovechar de este modo el tiempo en clase para desarrollar actividades de carácter más práctico (Bergmann \& Sams, 2012). Esta metodología puede sintetizarse en un antes y durante la clase (Angelini \& García-Carbonell, 2015) permitiendo al estudiante autorregular y autoplanificar su ritmo de trabajo, posibilita la profundización en temas de interés al estudiante bajo la supervisión docente, etc. (González \& Salcines, 2016).

Finalmente, esta metodología de enseñanza debe estar asociada a la adquisición de un conjunto de competencias profesionales genéricas y específicas (Tercer pilar) relacionadas con el ejercicio de la labor que el futuro graduado tendrá que desarrollar (Guerrero, 2011; Romero, Castejón, López- Pastor 6 Fraile, 2017).

Por todo ello, este planteamiento y orden de prioridad es lo que denominan Pérez-Pueyo et al, (2019) los tres pilares del proceso de transición pedagógica y didáctica. Como bien indican los autores las metodologías activas carecen de sentido si no se complementan con procesos de evaluación de carácter formativo. 
La presente experiencia de buena práctica está motivada por la necesidad de desarrollar $\mathrm{y}$ valorar nuevas experiencias en torno procesos evaluativos $\mathrm{y}$ metodológicos emergentes que permitan una adecuada adquisición de las competencias.

\section{Contextualización}

La experiencia se puso en práctica en la asignatura Evaluación y Recursos en la Enseñanza de la Educación Física del Máster de Formación de Profesorado. Han participado 23 estudiantes y dos profesores. En cuanto a las competencias profesionales que deben adquirir los estudiantes se pueden destacar (Figura 1):
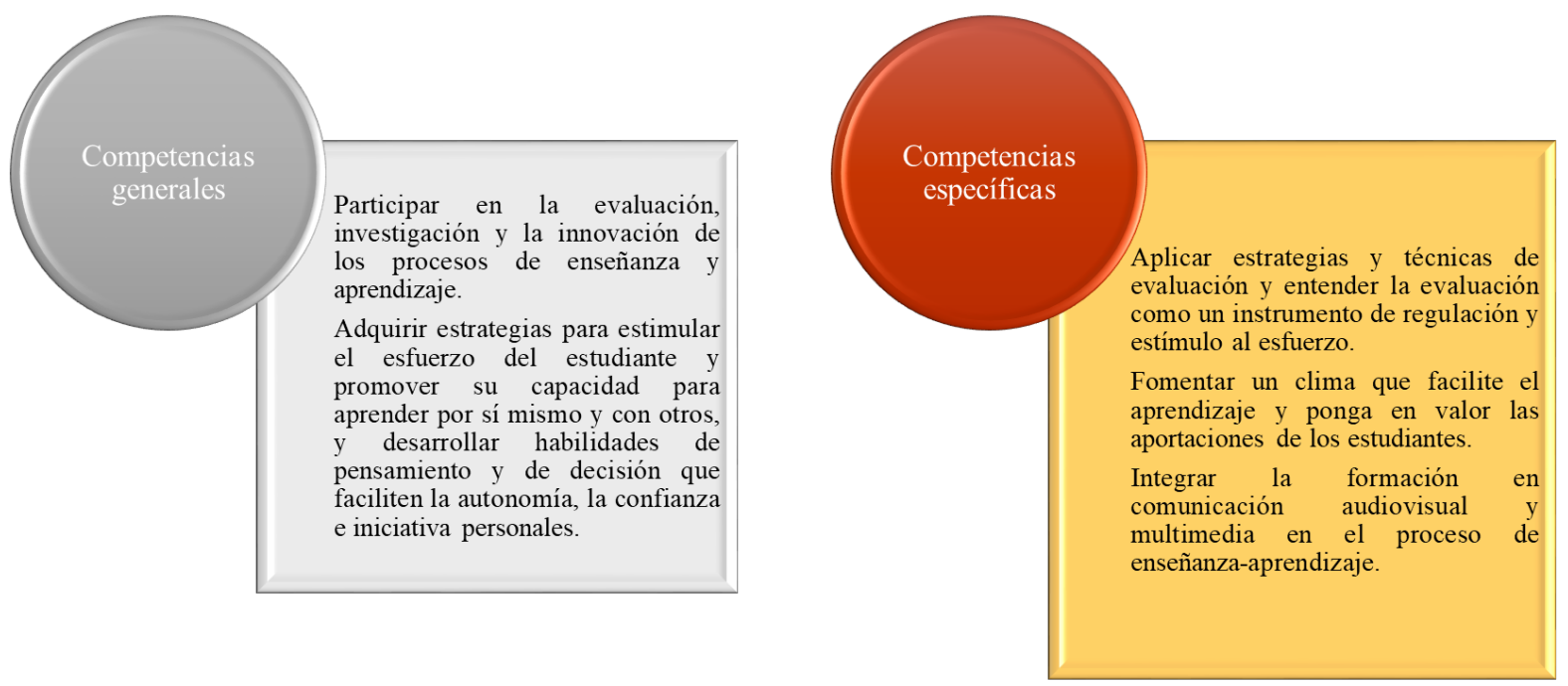

Figura 1. Competencias generales y específicas en el trabajo individual.

\section{Diseño y desarrollo}

Para llevar a cabo esta experiencia se planteó a los estudiantes la posibilidad de utilizar un proceso de evaluación formativa y compartida y una nueva metodología, frente a la clase tradicional. Esta dinámica de clases implicaba realizar cuatro tareas que formaban parte del trabajo individual (Figura 2). Se les informó que sería necesario un tiempo de trabajo en casa para revisar el material. Se comprometieron a trabajar en casa a través de un contrato didáctico (Blázquez, 2017) y a seguir un proceso con modalidad de evaluación formativa y continua. 


\begin{tabular}{|c|c|}
\hline $\begin{array}{l}\text { MODALIDAD A EVALUACIÓN A Y } \\
\text { METODOLOGÍA CLASE INVERTIDA }\end{array}$ & TRABAJO INDIVIDUAL \\
\hline \multicolumn{2}{|c|}{ PONDERACIÓN: 40\% (10\% cada una de las actividades) } \\
\hline \multicolumn{2}{|c|}{$\begin{array}{l}\text { CARÁCTER DE LA PRÁCTICA: realización de tareas formativas (clase invertida) sobre los vídeos, } \\
\text { documentos, blog, recursos, etc. Establecidos según temas de la asignatura }\end{array}$} \\
\hline \multicolumn{2}{|c|}{$\begin{array}{l}\text { ASPECTOS FORMALES: es individual y se realizará a ordenador. Cuidar mucho la presentación } \\
\text { (márgenes de } 3 \mathrm{~cm} \text {, letra Arial } 12 \text { e interlineado } 1.5 \text { ), la ortografía y la expresión }\end{array}$} \\
\hline \multicolumn{2}{|c|}{$\begin{array}{l}\text { PRESENTACIÓN DE LAS TAREAS: cada tarea se entregará en el aula virtual según fecha } \\
\text { establecida por el docente y consensuada con el estudiante (dos fases: fase previa con trabajo de casa y } \\
\text { fase supuesto práctico y evaluación con autoevaluación y autocalificación de dicha tarea) }\end{array}$} \\
\hline \multicolumn{2}{|c|}{$\begin{array}{l}\text { EVALUACIÓN: se realizará una evaluación compartida (previa autoevaluación) y calificación } \\
\text { dialogada (previa autocalificación) antes de la realización del examen. Las condiciones estarán } \\
\text { establecidas en el informe de autocalificación del trabajo individual. }\end{array}$} \\
\hline \multicolumn{2}{|c|}{$\begin{array}{l}\text { REQUISITOS: firmar y entregar el contrato didáctico, presentar las tareas según fechas establecidas y } \\
\text { asistencia }(85 \%) \text { con modalidad evaluación continua y formativa (Modalidad A) }(*) \text {. } \\
(*) \text { En el caso de presentar una actividad fuera de plazo se podrá presentar en la siguiente actividad } \\
\text { individual, pero tendrá un efecto directo en el criterio de calidad final de la actividad. En concreto se } \\
\text { restará un punto sobre el resultado final de dicha actividad. } \\
\text { La no entrega de uno de los trabajos y/o presencialidad implica el cambio en modalidad de evaluación. } \\
\text { La entrega del informe final de autoevaluación es un requisito. }\end{array}$} \\
\hline \multicolumn{2}{|c|}{ ESTRUCTURA QUE DEBE SEGUIR CADA TAREA: } \\
\hline \multicolumn{2}{|l|}{ Las tareas se estructura en dos fases: } \\
\hline Fase previa (casa) & a fin \\
\hline $\begin{array}{l}\text { El estudiante realizará la primera parte de la tarea } \\
\text { en casa (trabajo autónomo) y tiene que llevarla a } \\
\text { clase según fecha indicada por el docente en aula } \\
\text { virtual. }\end{array}$ & $\begin{array}{l}\text { a) Supuesto práctico: el estudiante, tutorizado por } \\
\text { el docente, elabora la segunda parte de la tarea en } \\
\text { clase. } \\
\text { Además, el docente dará un feed-back sobre la } \\
\text { primera parte de la tarea de cara a que el estudiante } \\
\text { pueda mejorar su calidad para la posterior entrega } \\
\text { definitiva } \\
\text { b) Evaluación: Autoevaluación- autocalificación } \\
\text { del estudiante (Rubrica de puntuación) }\end{array}$ \\
\hline $\begin{array}{r}* * * \text { Antes de la ejecución de estas tres tar } \\
\text { didáctico como proceso de aprendizaje y d }\end{array}$ & $\begin{array}{r}\text { e realizará una } \frac{T A R E A E J E M P L O}{\text { con el contrato }} \\
\text { aración de dudas. Esta tarea será evaluada pero no } \\
\text { calificada. }\end{array}$ \\
\hline TAREAS & PONDERACIÓN \\
\hline $\begin{array}{l}\text { Tarea ejemplo: elaboración de contrato didáctico } \\
\text { para ESO y/o Bachiller }\end{array}$ & Sin calificación \\
\hline $\begin{array}{l}\text { T1: Analizar experiencia de evaluación formativa } \\
\text { y/o compartida y elaborar un ejemplo con material } \\
\text { seleccionado por la docente }\end{array}$ & $10 \%$ \\
\hline $\begin{array}{l}\text { T2: Análisis LOMCE- ANOTA y supuesto de } \\
\text { unidad formativa con evaluación formativa y } \\
\text { compartida }\end{array}$ & $10 \%$ \\
\hline $\begin{array}{l}\text { T3. Análisis de Web interés con propuestas de } \\
\text { instrumentos de evaluación. Elaboración de una } \\
\text { rúbrica o escala de valoración }\end{array}$ & $10 \%$ \\
\hline T 4. Recursos & $10 \%$ \\
\hline
\end{tabular}
T 4. Recursos

Figura 2. Evaluación formativa y clase invertida para la elaboración del trabajo individual (Modalidad A evaluación formativa y compartida).

A continuación, se detalla un ejemplo explicando las dos fases.

Evaluación formativa y clase invertida para la adquisición de competencias en el Máster de Profesorado 
La fase previa (casa). Se enviaba al estudiante un documento con fecha de entrega antes del desarrollo del tema en clase presencial. El estudiante debía ver un vídeo, leer un documento de dos páginas y realizar una rutina de pensamiento. Finalmente debía indicar las dudas o preguntas sobre el tema trabajado (Figura 3).

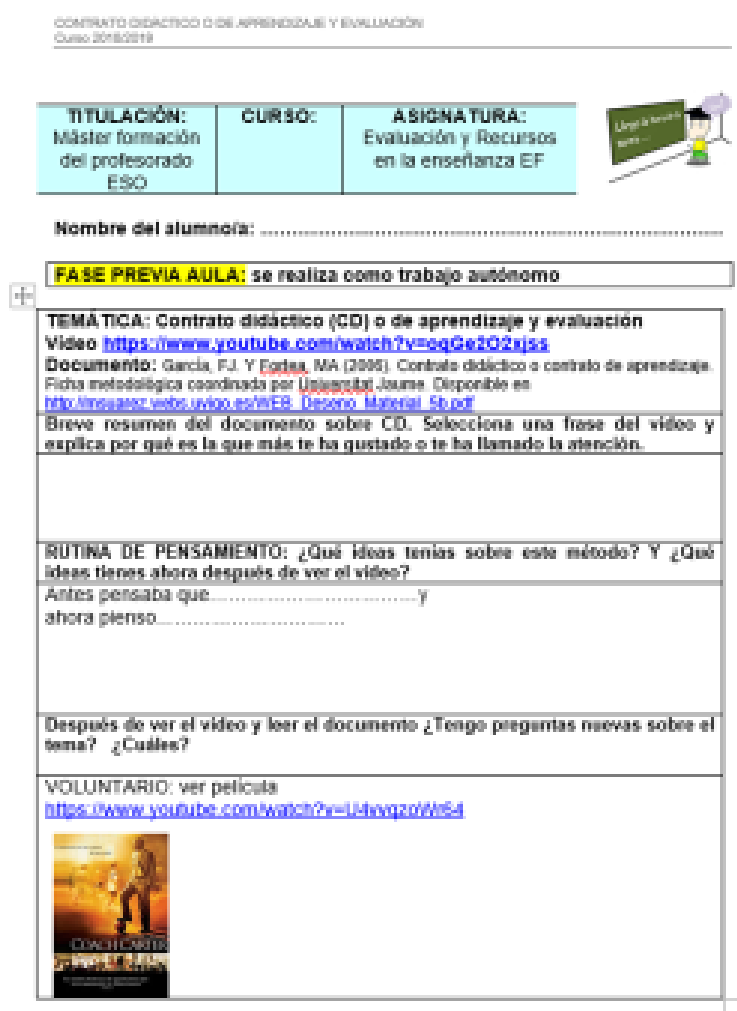

Figura 3. Fase previa en casa tarea trabajo individual.

Fase de aula (supuesto práctico y auto-evaluación y autocalificación) (Figura 2). La primera tarea era revisada por la docente y proporcionaba un feedback individual a cada estudiante. En el resto de las tareas la docente, en la clase presencial, proporciona a los estudiantes un feedback general a través de una evaluación formativa sobre el desarrollo de la tarea indicando puntos fuertes y débiles. Por tanto, la evaluación formativa se realiza dentro de los procesos de enseñanza y aprendizaje, con la intención de mejorar los propios procesos y a las personas implicadas en los mismos, con el propósito de analizar las causas de los posibles errores y abrir procesos de reflexión sobre los mismos, con el objeto de irlos superando y/o evitando (Herranz \& López Pastor, 2017). 
A continuación, se explicaban los contenidos esenciales, se dialogaba y se realizaba el supuesto práctico en el aula con ayuda de la docente (Figura 3). Finalizada la tarea, cada estudiante realizaba la autoevaluación de esta y se establece una nueva fecha de entrega de la tarea completa con las dos fases (previa y aula) y la evaluación de la misma (Figura 4).
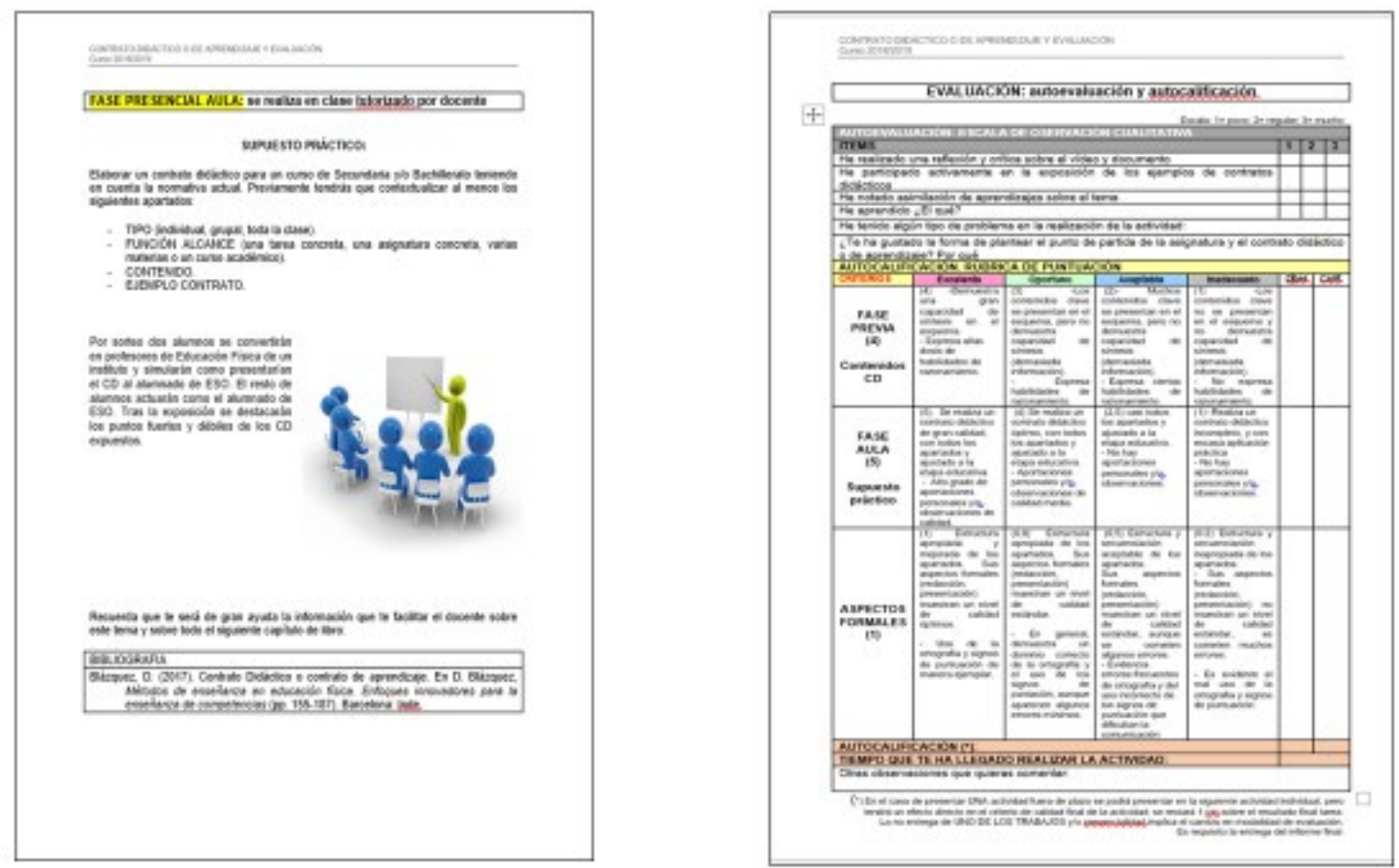

Figura 4. Fase aula final realizada en tiempo presencial.

Una vez finalizadas las cuatro tareas el estudiante tenía una semana de plazo para mandarlas de nuevo como portafolio completo incluyendo el autoinforme final. Esta experiencia de buena práctica fue evaluada por dos instrumentos estandarizados:

a. La Escala de Percepción sobre Metodologías EMPEF al finalizar la asignatura (Castejón, Santos, \& Palacios 2015).

b. La encuesta de satisfacción clase invertida de Gilboy, Heinerichs y Pazzaglia (2015) (https://goo.gl/forms/BIyYMHWSQnIDRHjj1).

\section{Evaluación y conclusiones}

Tras la puesta en práctica se considera que los estudiantes encontraron esta experiencia como muy positiva para su aprendizaje y rendimiento académico, al igual que otros estudios donde se utilizan metodologías activas y evaluación formativa y compartida 
(Barba-Martín \& López-Pastor, 2017). La tasa de éxito fue muy alta $(95,96 \%)$, con un alto rendimiento académico (34\% Sobresaliente, 55,52 \% Notable y 4,34\% Aprobado). Se consiguió que el estudiante tomase conciencia de sus progresos individuales en cada una de las tareas y se implicara en sus actividades de forma autónoma. Desde el punto de vista de la satisfacción se observó en la encuesta (ítems 2) que la mayoría de los estudiantes prefieren las actividades que se realizaron con clase invertida $(95,7 \%)$ en comparación a las que se realizan con clases tradicionales.

\section{Referencias}

Angelini, M. L. \& A. García-Carbonell (2015). Percepciones sobre la integración de modelos pedagógicos en la formación del profesorado: la simulación y juego y el flipped classroom. Education in the Knowledge Society, (16), 2,16-30. (http://revistas.usal.es/index.php/eks/article/view/eks20151621630/13502)

Arellano, N. M., Aguirre, J. F., \& Rosas, M. V. (2015). Clase invertida: una experiencia en la enseñanza de la programación. En X Congreso sobre Tecnología en Educación \& Educación en Tecnología (TE \& ET) (Corrientes, 2015).

Barrientos Hernán, E. J., López Pastor, V. M. \& Pérez Brunicardi, D. (2019) ¿Por qué hago evaluación formativa y compartida y/o evaluación para el aprendizaje en EF? La influencia de la formación inicial y permanente del profesorado. Retos, 36, 37-4 (https://recyt.fecyt.es/index.php/retos/article/view/66478)

Barba-Martín, R. \& López Pastor, VM (2017). Evaluación formativa y compartida en los proyectos de trabajo tutorizado, un ejemplo de buena práctica. Infancia, Educación $\quad y \quad$ Aprendizaje (IEYA), 2(3), 66-70. (http://revistas.uv.cl/index.php/IEYA/index)

Blázquez, D. (2017). Cómo evaluar bien Educación Física. El enfoque de la evaluación formativa. Barcelona: Inde.

Blasco, AC, Lorenzo, J. \& Sarsa, J. (2016). La clase invertida y el uso de vídeos de software educativo en la formación inicial del profesorado. Estudio cualitativo.@ tic. revista d'innovació educativa, 17, 12-20. (https://core.ac.uk/download/pdf/80524842.pdf)

Bergmann, J. \& Sams, A. (2012). Flip Your Classroom: Reach Every Student in Every Class Every Day. Washington, DC: International Society for Technology in Education. 
Castejón, F.J., Santos, M.L. \& Palacios, A. (2015). Cuestionario sobre metodología y evaluación en formación inicial en educación física. Revista Internacional de Medicina y Ciencias de la Actividad Física y el Deporte, (58), 15, 245-267. (http://cdeporte.rediris.es/revista/revista58/artescala566.htm)

Díaz, J.J.; Sánchez, M.M. \& I.M. Solano (2014). Metodologías activas con recursos audiovisuales en red: flippeando en clase. II Congreso Internacional de Innovación Docente, Murcia: Universidad de Murcia.

Gilboy, M. B., Heinerichs, S. \& Pazzaglia, G. (2015). Enhancing student engagement using the flipped classroom. Journal of Nutrition Educaction and Behavior, 47(1), 109-114

Guerrero, C. (2011). La evaluación del aprendizaje orientada a la evaluación por competencias en el Grado de Educación Social. RES Revista de Educación Social, 13, 1-17 (http://www.eduso.net/res/pdf/13/eval_res_13.pdf)

González-Fernández, N. \& Salcines, I. (2016). El modelo flipped classroom en el Grado de Educación Infantil. Una buena práctica en la Universidad de Cantabria. En A. Perez-Pueyo, MA Díez, C. Gutiérrez y D. Hortigüera, I Jornadas de buenas prácticas en Evaluación formativa en docencia (pp.100-125). León: Universidad de León.

Herranz, M. \& López Pastor, V. M. L. (2017). Perspectiva del alumnado sobre su participación en los procesos de evaluación formativa y compartida en educación física en primaria: Un estudio de caso. EmásF: revista digital de educación física, (48), 27-48 (http://emasf2.webcindario.com/EmasF_48.pdf)

Pérez-Pueyo, A., Casado, O., \& Hortigüela, D. (2019). La evaluación formativa, la autorregulación y la secuenciación de competencias. En J. Manso y J. Moya, Profesión y profesionalidad docente. Una acción educativa comprometida con el desarrollo humano (pp. 103-120). Andalucía: ANELE.

Manrique, J.C. (2016). El flipped classroom, un modelo pedagógico ideal para aplicar la evaluación formativa y compartida. En A. Perez-Pueyo, MA Díez, C. Gutiérrez \& D. Hortigüela, I Jornadas de buenas prácticas en Evaluación formativa en docencia (pp.259-265). León: Universidad de León. 
López-Pastor, V. M. \& Pérez-Pueyo, A. (2017). Evaluación formativa y compartida en educación: experiencias de éxito en todas las etapas educativas. León, España: Grupo IFAHE

Romero, M.R., Castejón, F.J., López, V.M. \& Fraile, A. (2017). Evaluación formativa, competencias comunicativas TIC en la formación del profesorado. Revista Comunicar, 52, 73-82. (https://doi.org/10.3916/C52-2017-07)

Trigueros Cervantes, C., Rivera García, E. (De la Torre Navarro, E. (2012). La evaluación en el aula universitaria: del examen tradicional a la autoevaluación. Revista Internacional de Medicina y Ciencias de la Actividad Física y el Deporte 12(47), 473-491. 